\title{
Terrorismo individual Individual terrorism
}

\author{
Alejandro Coteño Muñoz \\ Universidad Carlos III de Madrid \\ acoteno@pa.uc3m.es
}

Recibido / received: 29/06/2018

Aceptado / accepted: 16/07/2018

DOI: https://doi.org/10.20318/eunomia.2018.4356

\section{Resumen}

Los atentados perpetrados por actores solitarios en los últimos años han despertado el interés del legislador por la figura del terrorismo individual. No obstante, aunque este se regula en las últimas reformas penales, no se ha dispuesto una definición del mismo en términos positivos, sino que ésta únicamente se dispone en términos negativos con respecto al terrorismo colectivo. Así las cosas, en este trabajo se delinean los criterios para determinar cuándo un acto de terrorismo entra dentro del terrorismo individual y se aplican estas herramientas en tres casos paradigmáticos de lo generalmente conocido como "lobo solitario". Por último, se aportan tres clasificaciones diferentes del terrorista individual a fin de apuntalar una base sólida que permita una aproximación a sus distintos niveles de peligrosidad criminal.

\section{Palabras clave}

Terrorismo individual, organización terrorista, grupo terrorista, lobos solitarios, pertenencia.

\section{Abstract}

The attacks perpetrated on recent years by lone actors have aroused the interest of the lawmaker for the concept of individual terrorism. However, although it has been regulated in the last criminal reforms, a definition of it has not been positivized, a definition of it has not been provided in positive terms, but only in negative terms regarding collective terrorism. In this context, this study outlines the criteria to determine when an act of terrorism falls into individual terrorism and these tools are applied to three paradigmatic cases of what is generally known as lone wolves. Finally, three different classifications of the individual terrorist are provided to underpin a solid basis for an approach to their different levels of criminal dangerousness.

\section{Keywords}

Individual terrorism, terrorist organization, terrorist group, lone wolves, membership.

SUMARIO. 1. Introducción. 2. Origen y evolución del terrorismo individual. 3. El terrorismo individual como diferencia. 4. El terrorismo individual a través del fenómeno de los Lone wolves. 5. Tipos de terrorista individual, 5.1. La clasificación unifactorial de Pantucci, 5.2. La clasificación bifactorial del NSCITF, 5.3. La clasificación multifactorial de Bates. 6. Conclusiones. 


\section{Introducción}

La regulación penal del terrorismo ha pivotado tradicionalmente sobre la idea de la comisión de una serie de delitos con una finalidad política en el seno de un colectivo. De esta manera, se estimaba que los delitos terroristas únicamente podían ser perpetrados a través de una organización o banda armada. Debido a que el terrorismo es una de las mayores, sino la mayor, amenazas al Estado de Derecho a causa de su objetivo de subversión del orden constitucional, se consideraba que su especial peligrosidad radicaba, además de en su carácter político, en la actuación colectiva ${ }^{1}$.

Sin embargo, en los últimos veinte años, el Legislador, tanto nacional como internacional, ha comenzado a mostrar una gran preocupación por el terrorismo en su vertiente individual y así lo ha proyectado en su tipificación junto al terrorismo de carácter colectivo. Por un lado, en España, la sanción del terrorismo individual se plasmó en el art. $577 \mathrm{CP}^{2}$, de forma excepcional y con el objetivo de poder penar como terrorismo los desórdenes públicos realizados por la Kale Borroka, dado que su finalidad era coincidente a la de ETA (Cancio Melia, 2010: 263-266). Por otro lado, desde hace diez años, también se viene haciendo referencia a este tipo de terrorista, en su proyección yihadista, en los distintos textos penales en la materia ${ }^{3}$.

Como de estos dos ámbitos legales se deduce únicamente que el terrorismo individual está constituido por aquel terrorismo que no es en grupo, deviene inexcusable realizar un estudio detallado del concepto de "terrorismo individual". Así, a partir de este análisis se podrá establecer una base concreta para posteriores estudios donde se evalúe la capacidad de este tipo de terrorismo no organizado de lesionar y/o poner en peligro el bien jurídico protegido por este tipo de delitos.

Con este fin, en primer lugar, este trabajo estudia la evolución del terrorismo desde la Revolución Francesa hasta nuestros días, haciendo especial hincapié en la historia del terrorismo individual desde la "Propaganda por el hecho" anarquista. Posteriormente, se examina el significado penal del término a partir de la diferencia con respecto al terrorismo colectivo. De esta manera se consigue determinar qué casos podrán ser tratados como terrorismo colectivo y cuáles habrán de ser incardinados dentro del terrorismo individual.

Entonces, una vez que se hayan determinado los criterios para diferenciar estos dos tipos de terrorismo, se analiza el fenómeno de los "lobos solitarios". Para ello, se estudian las diferencias entre los casos que comúnmente son englobados dentro de este término, a saber: i) el caso del terrorista individual propiamente dicho; ii) el del terrorista individual táctico; y iii) el del lobo solitario yihadista. Además, con el

\footnotetext{
${ }^{1}$ Esto es así porque la adicional peligrosidad se justifica en el hecho de que lo injusto de la conducta colectiva supera a la mera suma de los injustos de las acciones individuales (Lampe, 2003).

2 Este tipo rezaba lo siguiente en su versión de 1995: "Los que, sin pertenecer a banda armada, organización o grupo terrorista, y con la finalidad de subvertir el orden constitucional o de alterar la paz pública, o la de contribuir a estos fines atemorizando a los habitantes de una población o a los miembros de un colectivo social, político o profesional, cometieren homicidios, lesiones de las tipificadas en los arts. 147 a 150, detenciones ilegales, secuestros, amenazas o coacciones contra las personas, o llevaren a cabo cualesquiera delitos de incendios, estragos, daños de los tipificados en los art. 263 a 266, 323 ó 560 , o tenencia, fabricación, depósito, tráfico, transporte o suministro de armas, municiones o sustancias o aparatos explosivos, inflamables, incendiarios o asfixiantes, o de sus componentes, serán castigados con la pena que corresponda al hecho cometido, en su mitad superior".

${ }^{3}$ Dos ejemplos al respecto pueden hallarse en la Resolución del Consejo de Seguridad de la ONU 2178 (2014), donde se hace referencia al riesgo que plantean los combatientes individuales procedentes de Oriente Medio, y en la DM 2008/919/JAI, en la que se destaca la necesidad de modificar las legislaciones nacionales a fin de hacer frente a los "nuevos grupos semiautónomos ligados entre sí con flexibilidad", en clara referencia a las células yihadistas, las cuales no parecen ostentar las relaciones de jerarquía del terrorismo colectivo. Estas células serán estudiadas más adelante dentro del terrorismo individual.
} 
objetivo de ahondar aún más en el análisis del terrorismo individual, se examinan los diferentes tipos de terroristas individuales que pueden existir según su conexión con diferentes grupos, su autonomía ideológica, su motivación, su modo de radicalización y su forma de actuar.

En último lugar, se aportan una serie de conclusiones que contribuyen a la formación de un concepto de "terrorismo individual", el cual supera su previa caracterización como diferencia respecto del terrorismo grupal o colectivo. De esta manera, el concepto adquiere una nueva dimensión positiva que permite incluir ciertas consideraciones sobre su peligrosidad criminal.

\section{Origen y evolución del terrorismo individual}

Aunque los expertos en la materia suelen hacer referencia a la "propaganda por el hecho" anarquista como origen del terrorismo individual, es necesario realizar una breve reflexión sobre la evolución del terrorismo desde su surgimiento, al menos terminológicamente, en la Revolución Francesa. Este reducido análisis histórico permitirá comprender tanto la creciente utilización de la violencia como herramienta de emancipación de las masas, como la perpetración de ataques de manera individual.

El concepto de terrorismo encuentra su punto de partida en la identificación del terror con la virtud y, por ende, en la utilización del terror como método expeditivo de justicia revolucionaria realizada por Robespierre ${ }^{45}$. De esta revolución surge la declaración del derecho de asilo para quienes huían "por defender la libertad y no a los tiranos" positivizado en la Constitución Francesa de 1793, pues ante los poderes absolutos del Antiguo Régimen, la figura del delincuente político adquiere un aura de nobleza y heroísmo que lo hacía merecedor no del castigo, sino de honores y del reconocimiento general (Montoro Ballesteros, 2000: 139).

Así las cosas, en el año 1815 el Reino Unido rechaza la extradición pasiva de delincuentes políticos (Jiménez de Asúa, 1958: 185) ${ }^{6}$. Más tarde, en 1831, Francia publica una Circular en la que se declara que los delitos políticos están excluidos de la extradición, a lo que le sigue la Ley Belga de 1833 consolidando esta doctrina. Esta cláusula sería incluida a partir de este momento en numerosos tratados franceses con otros Estados (Kinneally III, 1987: 207). En vista de ello, el delincuente político era considerado más un luchador por la libertad que un terrorista.

Sin embargo, esta postura sufriría un primer cambio mediante la Cláusula Belga del Atentado, incluida en el Tratado de Extradición suscrito por Francia y Bélgica en 1856. A causa de la negativa por parte de Bélgica a entregar a Felice Orsini, supuesto autor del atentado contra Napoleón III, se establece que no se excluirán de la extradición los delitos políticos de atentado contra el jefe de un Estado

\footnotetext{
${ }^{4}$ Robespierre (1794: 7-8) afirma que "si la fuerza del Gobierno popular es, en tiempo de paz, la virtud, la fuerza del Gobierno popular en tiempo de revolución es, al mismo tiempo, la virtud y el terror. La virtud, sin la cual el terror es cosa funesta; el terror, sin el cual la virtud es impotente. Es mucho menos un principio contingente, que una consecuencia del principio general de la democracia aplicada a las necesidades más urgentes de la patria". A mayor abundamiento, véase Perceval Verde (2017: 119-152) y Aguilar Blanc (2012).

${ }^{5}$ Si bien el terrorismo como concepto surge con la Revolución Francesa, el uso de la violencia con fines políticos tiene su origen muchos siglos antes. En este sentido, Miller (1995: 29) cita como ejemplos los tiranicidios y regicidios en la Antigua Grecia y Roma.

${ }^{6}$ La promulgación de esta Ley tiene su origen en el escándalo que provocó en el Reino Unido la extradición a España de varios emigrados políticos contrarios al rey Fernando VII. Para más información véase Orchard (2014: 85), Pyle (2001: 80) o Prakash Sinha (2013: 172).
} 
extranjero o su familia, siempre que éste revista la forma de homicidio, asesinato o envenenamiento (Capella i Roig, 2014: 12).

Este primer cambio de postura halla otra de sus justificaciones en el ascendente terrorismo nacionalista ejercido por los Carbonari en Italia ${ }^{7}$, como movimiento de liberación nacional italiana, a partir de 1807 (Miller, 1995: 32-34), y más tarde por los Fenianos en Irlanda, como autoafirmación gaélica, a partir de 1858 (Burleigh, 2013: 7-44).

Más allá de estos episodios de terrorismo con tintes nacionalistas, a partir de mediados del Siglo XIX se empieza a popularizar la concepción del empleo de la violencia como medio para conseguir la revolución popular. El primer exponente de esta postura es la obra Murder and Liberty (Asesinato y Libertad) de Heinzen. En ella, el autor expone que emplear la violencia para conseguir justicia no es moral, si bien en un mundo de opresión ésta se convierte, al fin y al cabo, en una herramienta de defensa propia ${ }^{8}$.

Al hilo de esta idea de la violencia como herramienta de emancipación surgen dos estrategias terroristas diferentes dentro de los movimientos revolucionarios. Por un lado, se encuentra el terrorismo socialista de carácter colectivo representado en una primera época por Narodnaya Volya (Voluntad del Pueblo) y, más tarde, por la facción de combate del Partido Socialdemócrata de Rusia. Este terrorismo tiene un carácter espontáneo, aspecto por el que ha sido ampliamente criticado desde las filas bolcheviques (Lenin, 1902: 150-155; Trotsky, 1935: 63-73), al igual que por su carácter minimizador de la lucha de clases y por su estímulo a la represión desde el poder gubernamental (Trotsky, 1914: 2-4).

Por otro lado, en contraposición al terrorismo de carácter grupal surgido en Rusia, nace la "propaganda por el hecho" anarquista, que constituye la primera teorización profunda del terrorismo individual. Es ampliamente aceptado que la esencia de esta utilización de la violencia como herramienta de emancipación social se halla en Nechaev y Bakunin ${ }^{9}$, si bien, Kropotkin (1914: 10 y ss.) también aporta un razonamiento muy claro al respecto, cuando afirma que un primer acto violento sirve de inspiración al resto debido a que el Gobierno lo reprimirá con dureza y será esta grave represión, efectivamente, la que dará alas al pueblo.

En relación con la noción del atentado político como estímulo para la reacción social, dos son las causas que expresan el motivo del nacimiento de esta propaganda por el hecho anarquista. En primer lugar, se consideraba que, ante la exacerbada analfabetización de las masas, era necesario algún acto concreto que, sin la necesidad de profundas teorías, permitiera demostrar al conjunto de la población que se podía y se debía luchar contra aquellos que les oprimían. A este respecto, el acto violento se manifiesta como propaganda y ejemplo de la validez y actualidad del ideario anarquista (Iviansky, 1977: 44-48).

En segundo lugar, las mejoras e innovaciones en el ámbito de la comunicación y el transporte permitían una mayor difusión de los hechos acaecidos en los grandes

\footnotetext{
${ }^{7}$ El propio Felice Orsini era miembro de la organización nacionalista italiana "Joven Italia" creada por Giuseppe Mazzini, antiguo miembro de los Carbonari.

${ }^{8}$ Citado en Miller (1995: 36-37).

${ }^{9}$ La vinculación de Nechaev (1868: 1-6) con el terrorismo se percibe por su defensa de la destrucción completa de todo el sistema, incluyendo obviamente a sus dirigentes. Bakunin (1870), por su parte, llama a los campesinos a levantarse contra todas las instituciones pues tanto su ejemplo como la posterior reacción represora del Estado crearán las condiciones para el surgimiento de mayores y más numerosas iniciativas de emancipación.
} 
núcleos de población. Así, en este momento, los ataques contra personalidades poderosas podían llegar a casi todo el país en unos pocos días (Rapoport, 2004: 4849). Por todo ello, este terrorismo anarquista funcionaba como estímulo a la actuación individual y como prueba de que una sola persona podía infligir un gran daño al sistema.

Este terrorismo individual de carácter anarquista sigue vigente en España durante las dos primeras décadas del siglo XX a través de grandes atentados, entre los cuales se pueden citar el perpetrado por Mateo Morral contra Alfonso XIII (1905), el atentado contra Canalejas (1912) y el perpetrado contra Eduardo Dato (1921). Así las cosas, tras este último atentado y debido a la represión practicada por la dictadura de Primo de Rivera la violencia anarquista decae, aunque sin llegar a desaparecer durante la década de los años veinte. Posteriormente, con la proclamación de la Segunda República, la acción anarquista pasa a orientarse hacia "huelgas revolucionarias" y no tanto hacia atentados contra la autoridad. Sin embargo, tanto la no aceptación de las propuestas de la CNT y la FAI, como el golpe de Estado de julio de 1936 y la posterior guerra civil, provocan la reaparición de la violencia revolucionaria anarquista (Casanova Ruiz, 2005: 89-96).

No obstante, este terrorismo anarquista poco a poco irá debilitándose, lo cual se debe, en España, a una mayor represión, y en el resto del mundo, a una más profunda reflexión estratégica dentro del anarquismo, a la victoria política del marxismo dentro de la izquierda y al reforzamiento de un anarquismo no violento gracias al Estado del bienestar (García Moriyón, 2017: 130 y ss.).

No será hasta los años $60^{10}$, y ya fuera del anarquismo, cuando vuelva a resurgir el terrorismo individual gracias a la teoría de la Leaderless Resistance (Resistencia sin Líderes). Este renovado terrorismo individual se integra dentro de la lucha anticomunista en Estados Unidos y en sus países aliados, y no se organiza de manera grupal debido a la enorme dificultad de la actuación colectiva ante la superioridad policial (Nesser, 2012: 1 y ss.). Como es obvio, la táctica de luchar de manera solitaria para no ser capturado existía desde mucho tiempo antes, pero es a partir de este momento cuando comienza a plantearse de forma explícita.

Esta teoría se populariza en las dos últimas décadas del Siglo XX en la lucha de los supremacistas estadounidenses contra las leyes antirracistas. Especialmente destacable por la claridad de su exposición es la afirmación de uno de sus ideólogos, Louis Beam (1992), relativa a que se deberá actuar mediante pequeñas células fantasma de como mucho seis miembros o de manera individual, sin un liderazgo central, pero siguiendo una ideología común.

Este mismo razonamiento será posteriormente seguido por Setmarian tras los atentados del 11-S para afirmar que, debido al incremento de la vigilancia estadounidense tras los mencionados ataques, se hacía necesaria una nueva estrategia basada en pequeñas células desconectadas 0 , incluso, en actores solitarios (Pérez Ventura, 2014: 23). No obstante, este ideólogo y líder yihadista identificó también la flexibilidad y el potencial captador de nuevos miembros como ventajas adicionales del terrorismo individual (Cruickshank y Ali, 2007: 9).

Finalmente, y aunque con el auge de DAESH el terrorismo de carácter colectivo ha afirmado de nuevo su ineludibilidad, el aumento de la vigilancia policial a

\footnotetext{
${ }^{10}$ En este periodo intermedio resulta realmente complejo encontrar ejemplos de terrorismo individual. Quizá el más destacado sea, aunque a finales de la década de los treinta, concretamente en 1938, el asesinato de Ernst vom Rath, diplomático alemán en París, a manos de Herschel Grynzspan (Associated Press, 2017: 26).
} 
nivel global y la pérdida de territorios por parte de éste en Siria e Irak están revitalizando la amenaza siempre presente del terrorismo individual. A este respecto, la gran mayoría de los expertos en la materia advierten del muy probable aumento del número de ataques realizados por lobos solitarios en Europa y Norteamérica ${ }^{11}$.

\section{El terrorismo individual como diferencia}

De este aumento de la vigilancia policial y de las predicciones de aumento del terrorismo individual surge la necesidad de definir este concepto desde el ámbito penal y, a partir de ahí, comenzar a evaluar diferentes aspectos tales como su potencialidad lesiva o su relación con el fenómeno de los lobos solitarios.

Para este propósito, toda vez que ni el Código Penal ni ningún texto penal internacional relativo al terrorismo ofrecen una definición del mismo que vaya allá de la mera referencia a la comisión del acto terrorista por una sola persona, debemos utilizar los conceptos que sí son definidos en estos textos a fin de realizar una primera aproximación a la modalidad individual de comisión de estos delitos.

Esta se va a llevar a cabo a partir del estudio de los conceptos de "organización terrorista" y "grupo terrorista", con el objeto de aportar una primera descripción del término desde la diferencia que mantiene con la modalidad colectiva de comisión de estos delitos. En otras palabras, todo lo que no sea cometer un delito de terrorismo como miembro de un grupo u organización será considerado como cometerlo de manera individual.

De acuerdo con el art. 570 bis CP se entiende por "organización criminal" la "agrupación formada por más de dos personas con carácter estable o por tiempo indefinido, que de manera concertada y coordinada se reparte tareas o funciones a fin de cometer delitos". Asimismo, en el art. 571 CP se especifica que serán consideradas organizaciones terroristas aquellas que cuenten con las características del art. 570 bis CP y tengan por finalidad las comprendidas en el art. 573.1 CP relativas a los delitos terroristas.

Por otro lado, el art. 570 ter 1 CP dispone que el "grupo criminal" se caracteriza por estar formado por más de dos personas, tener como fin la comisión de delitos y por no gozar de la permanencia y/o división de tareas de las que goza la organización criminal. A mayor abundamiento, el art. 3 de la Convención de Palermo define al "grupo estructurado" (para nosotros, grupo criminal) como un "grupo no formado fortuitamente para la comisión inmediata de un delito y en el que no necesariamente se hayan asignado a sus miembros funciones formalmente definidas ni haya continuidad en la condición de miembro o exista una estructura desarrollada". Análogamente al caso de la organización terrorista, el grupo terrorista será aquel que cuente con todas las características del grupo criminal y cometa delitos con una finalidad terrorista.

Del estudio de ambos términos se percibe que el grupo criminal se define eliminando la concurrencia de algunas de las características de la organización criminal. Es por ello que la definición de terrorismo individual debe derivarse de la supresión de algunos de los rasgos del grupo. Así, a simple vista se puede determinar que ningún colectivo formado únicamente por dos personas puede ser considerado ni organización ni grupo criminal, de manera que sus miembros deberán ser catalogados como terroristas individuales. Sin embargo, se deben estudiar los colectivos terroristas formados por más de dos personas a fin de determinar si se trata

\footnotetext{
11 Véase Toboso Buezo (2017) y EUROPOL (2016), entre otros.
} 
de una organización, un grupo o un simple supuesto de codelincuencia, según las circunstancias del caso.

Aunque, como ya hemos visto, el Código Penal define con aparente claridad el grupo criminal a partir de la organización, cuando la Jurisprudencia se plantea la diferencia entre el grupo y la codelincuencia, la caracterización del grupo ubicada en el art. 570 ter 1 CP deviene demasiado simple y contradictoria. En este sentido, tres son los factores problemáticos que se han identificado a la hora de establecer esta diferenciación: i) la pluralidad delictiva; ii) la permanencia; y iii) la estructura del grupo criminal.

Para comenzar, la pluralidad delictiva se contempla en la propia definición de "organización criminal" al exigirle a ésta el fin de "cometer delitos". En este sentido, ante las divergencias que han surgido a la hora de interpretar esta finalidad, la Jurisprudencia mayoritaria ${ }^{12}$ ha afirmado que es ineludible que se cometan dos o más delitos, o que se pretenda su comisión, para que se pueda hablar de grupo criminal.

Sin embargo, la Jurisprudencia minoritaria ${ }^{13}$ ha entendido la pluralidad del término "delitos" del art. 570 ter 1 CP como la posibilidad de comisión de diferentes delitos. Dicho de otro modo, entiende la pluralidad en su carácter cualitativo y no cuantitativo. Esta argumentación gana peso si se atiende a una pequeña modificación del Código Penal realizada en la última reforma de 2015, a saber, la eliminación de la referencia a la "comisión reiterada de delitos", dejando simplemente el término "delitos" en plural. No obstante, esta interpretación teleológica no ha sido seguida por la Jurisprudencia tras dicha reforma, pues en las SSTS 277/2016 de 6 de abril (RJ $10714 / 2015, \mathrm{FJ} \mathrm{5}^{\circ}$ ) y 607/2017 de 15 de septiembre (RJ 10792/2016, FJ $3^{\circ}$ ), entre otras, se exige explícitamente una pluralidad en sentido cuantitativo.

Entonces, ahora que ha quedado demostrado que la pluralidad delictiva ha de entenderse de forma cuantitativa, resta analizar los otros dos factores problemáticos, así como la relación entre ellos, pues, aunque el texto penal permite incluso la no concurrencia de ambos para hablar de grupo criminal, la realidad resulta algo más complicada ${ }^{14}$.

En cuanto a la permanencia, primero se necesita definir qué se entiende por "fortuito", pues numerosas sentencias hacen referencia a la necesaria concurrencia de una "mínima permanencia" del grupo criminal a fin de poder afirmar su necesario carácter no fortuito, todo ello de acuerdo con el art. 3 de la Convención de Palermo ${ }^{15}$. Así las cosas, aunque la Doctrina y la Jurisprudencia suelen echar mano de este concepto sin concretarlo, existe una resolución donde se le define, la STS 798/2016 de 25 de octubre (RJ 10224/2016, FJ 1º). Esta sentencia caracteriza el término

\footnotetext{
12 Véase SSTS 798/2016 de 25 de octubre (RJ 10224/2016, FJ $1^{\circ}$ ), 714/2016 de 26 de septiembre (RJ 1951/2015, FJ 12 ${ }^{\circ}$ ), 277/2016 de 6 de abril (RJ 10714/2015, FJ 5º), 576/2014 de 18 de julio (RJ $10028 / 2014$, FFJJ $2^{\circ}, 3^{\circ}$ y $6^{\circ}$ ) y $577 / 2014$ de 12 de julio (RJ $10071 / 2014$, FJ $7^{\circ}$ ), entre otras.

${ }^{13}$ Véase SSTS 289/2014 de 8 de abril (RJ 2158/2013, FJ $8^{\circ}$ ) y 445/2014 de 29 de mayo (RJ 11106/2013, FJ $14^{\circ}$ ), entre otras.

${ }^{14}$ En este sentido, la apreciación únicamente de pluralidad delictiva resulta insuficiente para caracterizar al grupo criminal al no permitir esta, por si sola, la diferenciación de este respecto de la codelincuencia, dado que cabe concebir un colectivo formado fortuitamente que comete en un lapso breve de tiempo una serie de delitos en concurso real.

15 Véase SSTS 576/2014 de 18 de julio (RJ 10028/2014, FFJJ $2^{\circ}, 3^{\circ}$ y $6^{\circ}$ ), 577/2014 de 12 de julio (RJ 10071/2014, FJ $7^{\circ}$ ), 309/2013 de 1 de abril (RJ 11151/2012, FJ 2º, 714/2016 de 26 de septiembre (RJ 1951/2015, FJ 12\%), 277/2016 de 6 de abril (RJ 10714/2015, FJ 5'), 719/2013 de 9 de octubre (RJ

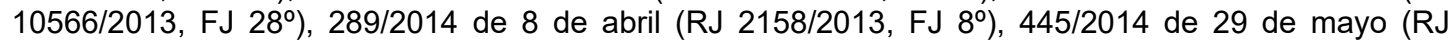
$11106 / 2013$, FJ $14^{\circ}$ ), 271/2014 de 25 de marzo (RJ 10892/2014, FJ $4^{\circ}$ ).
} 
"fortuito" como un "suceso inopinado o casual, fruto de una decisión inmediata, repentina o proyectada a la comisión de una acción concreta".

Por tanto, de acuerdo con el razonamiento expresado repetidamente por el Alto Tribunal, el carácter fortuito operaría como el contrario a la permanencia, siendo así que se acreditaría la existencia de una mínima permanencia en el momento en el que la comisión de los delitos requiera cierta planificación y no se trate de una decisión inmediata o repentina.

Finalmente, la Jurisprudencia ha identificado en varias sentencias la referencia a la perpetración "concertada" de delitos del art. 570 ter 1 CP como la necesidad de que el grupo criminal goce de una mínima estructura ${ }^{16}$. En este sentido, cabe destacar la SAN 70/2013 de 20 de diciembre (RJ 5522/2013, FJ $2^{\circ}$ ) que, ubicando al grupo criminal en un estadio intermedio entre la organización criminal y la codelincuencia y tomando como requisito la pluralidad delictiva, afirma que es necesario una mínima estructura que permita, al fin y al cabo, proyectar esa pluralidad delictiva más allá del mero consorcio fortuito entre más de dos personas.

Dicho esto, surge una contradicción. Mientras que el Código Penal no exige ni una mínima permanencia ni una mínima estructura, pues con la concurrencia de más de dos personas y la finalidad de comisión de delitos se podría hablar de grupo criminal, la Jurisprudencia afirma la necesidad de una mínima permanencia y de una mínima estructura. En otras palabras, mientras que el texto penal permite obviar dos de los requisitos de la organización criminal, la Jurisprudencia parece exigirlos, aunque en un nivel inferior al de la organización.

Al fin y al cabo, una interpretación gramatical nos llevaría a aceptar la previsión del último párrafo del art. 570 ter $1 \mathrm{CP}$, si bien, ésta entraría en contradicción con la referencia del Legislador expresada en el Preámbulo de la LO 5/2010 de modificación de la LO 10/1995 del Código Penal relativa al plus de peligrosidad del grupo criminal con respecto a las acciones individuales de sus miembros, entendida ésta mayor peligrosidad como esencia del mismo. $Y$ es que este plus al que apuntamos hace referencia a la creación de sinergias de peligrosidad y no parece posible afirmar esta peligrosidad no exigiendo una mínima permanencia y/o estructura. Son estos dos factores los que permiten elevar una asociación de personas por encima de una simple unión fortuita.

En todo caso, y alejándonos de una interpretación gramatical a fin de poder establecer una diferencia clara entre el grupo criminal y la codelincuencia, resta examinar la forma de exigencia tanto de la mínima permanencia como de la mínima estructura, esto es, determinar si han de concurrir juntas o si, por el contrario, basta con la concurrencia de una de ellas para afirmar la existencia de un grupo criminal.

Con este objetivo, en primer lugar, y pese a que en la mayoría de los casos los conceptos "fortuito", como negación de la mínima permanencia, y "consorcio", como contrario a la mínima estructura, parecen encontrarse muy cercanos, no podemos determinar que la permanencia y la estructura deban concurrir juntos para hablar de grupo criminal. Esto es así por dos razones.

Por un lado, es perfectamente concebible un caso de comisión de delitos de manera reiterada por más de tres personas sin una distribución de tareas mínima, es

\footnotetext{
16 Véase SSTS 445/2014 de 29 de mayo (RJ 11106/2013, FJ $14^{\circ}$ ) y 289/2014 de 8 de abril (RJ $2158 / 2013$, FJ $8^{\circ}$ ). Aunque estas resoluciones acuden a la estructura habiendo negado previamente el requisito de la pluralidad, resultan de interés en atención al razonamiento sobre la necesaria mínima estructura.
} 
decir, realizándose las tareas organizativas de manera indistinta por los diversos coautores. En este caso, resulta claro que es esa mínima permanencia la que afirma el plus de peligrosidad necesario para hablar de grupo criminal y no de codelincuencia.

Análogamente, la estructura no siempre conlleva una mínima continuidad, por cuanto se puede imaginar el caso de un conjunto de personas que cometen dos delitos en un lapso breve de tiempo, bajo una estructura determinada y más o menos férrea. Aquí, entonces, es la propia estructura, la división de tareas, la que aporta el plus de peligrosidad requerido.

En consecuencia, es la necesidad de diferenciar el grupo criminal de la codelincuencia de acuerdo con su ubicación en un punto medio entre esta última y la organización criminal la que permite afirmar que el grupo criminal exige una mínima permanencia y/o estructura, siempre, como es lógico, en un nivel inferior a la organización criminal y sin que éstas deban concurrir de forma conjunta ${ }^{17}$.

Por tanto, hablaremos de terrorismo individual en los siguientes casos. Primero, siempre que se trate de un colectivo formado por menos de tres personas, pues con menos de este número no se alcanza a integrar la colectividad terrorista. Segundo, cuando el colectivo, goce del número de miembros que goce, busque cometer un solo delito terrorista. Tercero, cuando el colectivo de más de tres personas busque cometer una pluralidad de delitos sin mostrar ninguna estructura ni continuidad $^{18}$. Por el contrario, en el momento en que un colectivo de más de dos personas, buscando cometer más de un delito, proyecte una mínima estructura 0 permanencia en el tiempo, nos hallaremos ante un grupo terrorista.

\section{El terrorismo individual a través del fenómeno de los Lone wolves}

Ahora que ya se ha determinado en qué casos se puede hablar de terrorismo individual, se van a analizar las diferentes situaciones que generalmente se incluyen dentro de los casos de lone wolves o "lobos solitarios", lo cual nos servirá para profundizar algo más en el concepto de "terrorismo individual". En este epígrafe se determinarán las diferencias penales entre los tres casos paradigmáticos dentro de este fenómeno, a saber: i) el terrorista individual propiamente dicho; ii) el terrorista individual táctico; y iii) el comúnmente conocido como lobo solitario yihadista ${ }^{19}$. Además, para ello se repasarán los conceptos de pertenencia y colaboración.

Dentro de todo este fenómeno, el primer problema con el que nos encontramos es la confusión entre el "terrorista individual propiamente dicho" y el terrorista que actúa solo por motivos operativos o estratégicos pero que realmente pertenece o colabora con una organización, el cual ha venido a denominarse "terrorista individual táctico" (Toboso Buezo, 2014b: 101).

Esta confusión se produce debido al ya referenciado cambio de estrategia adoptado por el terrorismo yihadista tras los atentados del 11-S como consecuencia de la defensa que realiza Setmarian de la acción solitaria e individual. De esta manera, el terrorismo yihadista pasa a la orientarse hacia la lucha individual, provocando que

\footnotetext{
17 Todo ello teniendo en cuenta que de acuerdo con el texto penal en su literalidad no se exige ni permanencia ni estructura.

${ }^{18}$ En este caso, nos encontraremos, casi con toda seguridad, ante un supuesto de delito continuado.

${ }^{19}$ El término "lobo solitario", como a continuación se va a explicar, es usado por la doctrina anglosajona para referirse al terrorista individual puro. No obstante, aquí se usa la expresión "lobo solitario yihadista" para referirse a este tercer supuesto, aunque pueda crear confusión, debido a que así es como periodísticamente se le ha denominado ante la ausencia de otras denominaciones.
} 
la línea entre el terrorista individual y el terrorista que actúa en solitario por meras razones operativas se haga cada vez más difusa.

Debido a la escasez de estudios en el Derecho penal sobre esta específica modalidad de comisión de delitos terroristas, resulta necesario acudir a perspectivas policiales y securitarias a fin de examinar qué criterios son válidos para definir al terrorista individual propiamente dicho y así poderlo distinguir con claridad del terrorista individual táctico.

Para ello, se debe acudir a lo que la mayoría de los expertos anglosajones han denominado lone wolf. A este respecto, una de las definiciones más completas es la aportada por Spaaij (2010: 856), quien afirma que las características de este son las siguientes: i) opera individualmente; ii) no pertenece a ningún grupo, organización o red terrorista; y iii) su modus operandi es concebido por él mismo y no acata jerarquías, ni órdenes directas. Asimismo, también es citada por muchos la propuesta de Burton y Stewart, que dispone que el lobo solitario (para nosotros, el terrorista individual en términos estrictos) es "aquella persona que actúa por su cuenta sin recibir órdenes o siquiera tener conexiones con una organización” (2008: 5).

Si bien el requisito de la actuación individual, en el sentido de no organizada, se puede eliminar fácilmente por la posibilidad de actuación conjunta de dos personas sin que se pueda considerar organización o grupo terrorista, la ausencia de conexiones con una organización merece una reflexión más profunda. A este respecto, dos son las interpretaciones mayoritarias. De una parte, Toboso Buezo (2014a: 10) afirma que el terrorista individual no puede haber mantenido ningún contacto, siquiera histórico, con una organización terrorista, sino que debe haberse autorradicalizado. De otra parte, el Comité de Expertos en Terrorismo del Consejo de Europa (en adelante, CODEXTER) (CODEXTER, 2015: 3 y ss.) afirma que la clave se halla en la ausencia de recepción por parte del terrorista individual de órdenes directas de la organización o grupo criminal.

Para optar por una interpretación u otra resultan muy útiles los criterios expresados por la Jurisprudencia, concretamente en la STS 886/2007 de 2 de noviembre (RJ 10272/2007, FJ $1^{\circ}$ ), a la hora de determinar la pertenencia de un sujeto a una organización o grupo. Dichos criterios son: i) existencia objetiva de una organización o banda armada existiendo entre los miembros una coincidencia ideológica y un sometimiento a relaciones jerárquicas y de subordinación ${ }^{20}$; ii) una voluntad de pertenencia a la organización por parte del integrante con carácter permanente o indefinido, pero nunca de manera episódica; y iii) la realización o posibilidad de realización de actividades propias de la banda armada.

En este sentido, hemos de optar por el criterio mantenido por CODEXTER. En esencia, si no se reciben órdenes es porque no se está sujeto a ninguna jerarquía o subordinación, con lo que no puede apreciarse pertenencia. Si los contactos han sido mantenidos en el pasado, pero en el momento de comisión del delito terrorista no se aprecian, no se dan las condiciones necesarias para afirmar que el sujeto terrorista pertenezca a un colectivo terrorista.

Es más, aun cuando el contacto fuera actual, deberá estudiarse caso por caso si se cumplen los requisitos de la pertenencia. Esto es, se deberá comprobar si el sujeto se halla subordinado a los líderes o mandos intermedios de la organización, si

\footnotetext{
${ }^{20}$ Por esta razón, el terrorismo anarquista no puede llegar nunca a ser colectivo. Al fin y al cabo, no existe jerarquía en su actuar.
} 
su integración es permanente o indefinida y si, además, se encuentra en condiciones de realizar actividades propias de la banda.

Por el contrario, el terrorista individual táctico, de acuerdo con Pantucci, quien para el caso hace referencia al terrorismo yihadista y lo denomina lone attacker, será "aquel individuo que opera solo, pero que demuestra claramente que sigue órdenes o que está sometido a nexos de control por parte de la matriz de Al Qaeda o de otros grupos afiliados a esta" (2011). De esta manera se puede ver cómo, a través de las órdenes y del control por parte del colectivo terrorista, este sujeto sí que manifiesta pertenencia a la organización por lo que no puede ser, en ningún caso, considerado terrorista individual.

En conclusión, el aspecto clave que diferencia al terrorista individual propiamente dicho del terrorista individual táctico es el hecho de que el segundo sí que forma parte de una organización o grupo terrorista y actúa en soledad únicamente debido a razones operativas y/o estratégicas. Así, este está sujeto a una jerarquía y a una permanencia a las que el terrorista individual no tiene que hacer frente.

Entonces, ahora que ya se ha determinado que el primero de los casos a estudiar debe categorizarse como terrorismo individual, mientras que el segundo entra dentro del terrorismo a nivel colectivo, resta analizar un caso intermedio, el del lobo solitario yihadista.

Este es el caso de aquel terrorista que perpetra un atentado siguiendo la finalidad política de una determinada organización terrorista, en este caso DAESH, y obedeciendo, además, a una serie de directrices dictadas por los líderes de la misma a todos los potenciales individuos que pudieran ayudarles con sus propósitos. Se trata de un supuesto donde los líderes de la organización llaman a atentar contra Occidente a todos los comprometidos con la causa y, como consecuencia de ello, una persona que no tiene ninguna vinculación con el colectivo terrorista lleva a cabo un ataque.

Tal y como se percibe a primera vista, nos hallamos ante un supuesto intermedio entre el terrorista individual puro que atenta de acuerdo con sus propios objetivos políticos y el terrorista individual táctico que actúa en solitario únicamente por razones tácticas u operativas. Para determinar su inclusión dentro del terrorismo individual o del terrorismo colectivo, se analizará su relación con la organización terrorista subyacente a través de los conceptos de pertenencia y colaboración.

En primer lugar, para constatar su no pertenencia a la organización, es necesario acudir de nuevo a la STS 886/2007 de 2 de noviembre (RJ 10272/2007, FJ $\left.1^{\circ}\right)$. Para comenzar, fácilmente se verifica la existencia de una organización terrorista, en este caso DAESH, que es quien realiza dichos llamamientos a atentar en Internet. También se afirma sin mayores dificultades la posibilidad del lobo solitario de realizar acciones propias de la banda armada, entendiendo el asesinato de infieles como una actividad ordinaria de la organización en aras a la consecución de sus fines políticos.

Sin embargo, no es posible determinar la existencia de una relación jerárquica o de subordinación entre el lobo solitario y el líder o los mandos intermedios de la organización, ya que la única indicación o pauta que recibe el individuo es la de matar personas en países occidentales, sin mediar ninguna instrucción concreta para la realización de dicha actuación terrorista, y siendo además una directriz dirigida a un público de potenciales yihadistas amplísimo.

De la misma manera, el lobo solitario tampoco tiene, debido a las circunstancias de su actuación, una voluntad de actuar para la organización más allá 
de en un episodio concreto. Al tratarse de operaciones de inmolación o con un alto riesgo de ser asesinado por la Policía, el individuo terrorista no puede orientar su voluntad, de facto, a la realización de acciones de la organización de modo reiterado.

En segundo lugar, tampoco es posible afirmar la colaboración como criterio explicativo de la actuación de los lobos solitarios en relación con la organización terrorista DAESH. En este sentido, la negación de la existencia de colaboración, tanto en su vertiente material como en relación con las finalidades de la banda, tiene su origen en el hecho de que la actuación llevada a cabo por el lobo solitario no puede ser considerada periférica.

A efectos de determinar las actuaciones que deben ser consideradas periféricas dentro de una organización terrorista, se ha de acudir, en primer lugar, al art. $577 \mathrm{CP}$, donde se enumeran las siguientes a modo de ejemplo: "información o vigilancia de personas, bienes o instalaciones, la construcción, acondicionamiento, cesión o utilización de alojamientos o depósitos, la ocultación, acogimiento o traslado de personas, la organización de prácticas de entrenamiento o la asistencia a ellas, la prestación de servicios tecnológicos, y cualquier otra forma equivalente de cooperación o ayuda a las actividades de las organizaciones o grupos terroristas, grupos o personas a que se refiere el párrafo anterior".

Esta equivalencia a la que se refiere el legislador debe ser interpretada teleológicamente a fin de incluir dentro de la colaboración únicamente las actividades que contribuyan a reforzar las actuaciones de la organización y excluir, por otra parte, la comisión de delitos graves, que debe ser considerada la actividad principal a través de la que la banda armada intenta causar terror y subvertir el orden constitucional ${ }^{21}$.

La misma interpretación se realiza por la Audiencia Nacional en su SAN 28/2000 de 10 de julio (RJ 4836/2000, FJ $3^{\circ}$ ) al disponer que el delito de colaboración solo podrá apreciarse cuando la actividad realizada sea autónoma e independiente de cualquier otra actividad delictiva a la que haya contribuido, siendo así que la colaboración exige que el acto en sí de colaborar no se halle causalmente conectado a la producción de un resultado delictivo concreto.

Por tanto, la comisión de un delito grave, al constituir el medio principal de la organización terrorista para la consecución de sus fines políticos, no podrá ser considerada constitutiva de un delito de colaboración con organización terrorista. En cierto modo, esta actuación se hallaría más cerca de la pertenencia, si bien, esta queda excluida, como se ha explicado previamente, por la actuación esporádica del lobo solitario y por el desconocimiento de su integración en la banda armada por parte de los integrantes de la misma.

Por todo ello, se determina que la conducta del lobo solitario yihadista se debe incardinar dentro del terrorismo individual. La ausencia de conexiones, en el sentido de recepción de órdenes, al tiempo de comisión del delito con colectivos terroristas y el vínculo ideológico como único enlace con estos eliminan cualquier posibilidad de que se trate de un caso de pertenencia o colaboración.

\footnotetext{
${ }^{21}$ En este sentido se expresan Capita Remezal (2008: 143) y Cancio Melia (2010: 240) al afirmar que las conductas de colaboración son actividades que se identificarían en esencia con comportamientos propios de la complicidad, es decir, de actuación para la organización y no de intervención en la misma. A mayor abundamiento, comparto también en su totalidad lo defendido por Lamarca Pérez (1985: 282) relativo a que los colaboradores favorecen la acción organizativa y no la comisión de delitos, aunque en la práctica resulte harto difícil probar la diferencia.
} 
En conclusión, tanto el terrorista individual propiamente dicho como el lobo solitario yihadista se incluyen en el terrorismo individual. Por contra, el terrorista individual táctico sí que cumple con los requisitos expresados por la Jurisprudencia para pertenecer a una organización terrorista, por lo que se trata de un caso de terrorismo colectivo.

\section{Tipos de terrorista individual}

Más allá del estudio de la regulación penal relativa al terrorismo colectivo y del fenómeno de los lobos solitarios, y con el objeto de comprender todas las aristas del terrorismo individual, deben analizarse algunas de las clasificaciones que diferentes expertos han propuesto al efecto. Estas permitirán sentar las bases para la realización, en otros trabajos, de una evaluación del riesgo que cada uno de estos actores individuales representan y, con ello, de su potencialidad lesiva ${ }^{22}$.

Aunque la Doctrina no les ha otorgado las denominaciones que a continuación se utilizan, el hecho de que a medida que se avanza en el texto se vayan incluyendo nuevos factores o variables a tener en cuenta ha determinado que las diversas clasificaciones se nombren según el número de variables que incluyan. Así, en primer lugar, se analizará la clasificación unifactorial de Pantucci; después, la bifactorial del National Security Critical Issues Task Force (en adelante, NSCITF); y, en último lugar, la multifactorial de BATES ${ }^{23}$.

\subsection{La clasificación unifactorial de Pantucci ${ }^{24}$}

La primera de las clasificaciones a analizar es la postulada por Pantucci (2011). Es la más sencilla de comprender debido a que únicamente trata el nivel de adscripción o conexión del actor solitario con un colectivo terrorista, siendo este de ideología yihadista. Así, los tipos de terrorista individual propuestos por este autor son los siguientes: i) loner; ii) lone wolf; iii) lone wolf pack; y iv) lone attacker. A continuación, se realizará un análisis de cada de ellos.

El primero de ellos, el loner, es un actor solitario que, bajo el paraguas de la ideología yihadista, pero sin mantener ningún tipo de contacto con ningún miembro de ningún colectivo terrorista, ni siquiera con un simple extremista, perpetra un atentado. En este sentido, tres son sus rasgos característicos: i) se trata de un actor completamente solitario; ii) se halla ideológicamente fanatizado; y iii) no mantiene ningún vínculo con terceros.

En segundo lugar, elevando el nivel de contacto con un colectivo terrorista, se encuentran los lone wolf. Pantucci los define como "actores individuales que, mientras que parece que llevan a cabo sus acciones solos y sin ninguna conexión externa, de hecho, muestran cierto nivel de contacto con extremistas operativos" (ibídem: 22). En todo caso, para considerar que son terroristas individuales y no miembros de una organización terrorista, dichas conexiones no deben implicar, en modo alguno, jerarquía. De esta manera, si dichos vínculos únicamente se proyectan en inspiración

\footnotetext{
22 Es necesario apuntar que estas clasificaciones se han realizado desde fuera del derecho penal, pues dentro de esta rama del Derecho únicamente se concibe al terrorismo individual como el opuesto al terrorismo colectivo.

${ }^{23}$ Las denominaciones que cada uno de estos autores otorgan a los diferentes tipos de terrorista individual no han sido traducidas debido a que solo causarían confusión, dada su cercanía lingüística, así como debido a la usual confusión en torno al término "lobo solitario".

${ }^{24}$ Aunque este autor sólo hace referencia al terrorista yihadista, su clasificación puede proyectarse al común de los terroristas individuales.
} 
y adiestramiento o entrenamiento pasado, el lone wolf podrá ser considerado terrorista individual.

Tercero, como proyección de la unión de varios lone wolf, se hace referencia a los lone wolf pack. En palabras del autor, estos son "grupos de individuos que se autorradicalizan usando la narrativa de Al-Qaeda" (ibídem: 24). Las características del lone wolf son perfectamente aplicables a este grupo debido a que sus conexiones con colectivos yihadistas no podrán implicar pertenencia o colaboración.

En este ámbito, surge la duda de si todos los lone wolf pack pueden ser considerados ejemplos de terrorismo individual. Aunque Pantucci los cataloga como una simple extensión del lone wolf, en aplicación de lo explicado en el epígrafe tercero, únicamente podrá ser incluidos dentro del terrorismo individual si se hallan formados por dos personas, o incluso tres, siempre que se orienten a la comisión de un único delito o no muestren ni una mínima continuidad ni una mínima estructura.

Finalmente, como máxima expresión de la conexión con un grupo terrorista, el autor hace referencia al lone attacker. Como se ha explicado en el epígrafe anterior, éste es perfectamente equiparable con lo que en la doctrina española se ha venido a denominar "terrorista individual táctico". En este sentido, como sigue órdenes y/o se halla controlado por el colectivo terrorista, se entiende que se trata de un ejemplo de pertenencia y no de terrorismo individual.

En consecuencia, gracias a los diferentes niveles de conexión con colectivos terroristas que estos tipos de terrorista individual muestran, se puede afirmar que el que proyecta una mayor peligrosidad, exceptuando al lone attacker, es el lone wolf pack, debido a la pluralidad de sujetos implicados y por contar con cierto contacto con una organización o grupo terrorista. Por otro lado, debido también a este superior contacto con un colectivo del lone wolf con respecto al loner, es lógico pensar que el primero representa una mayor peligrosidad, pues se hallará en unas condiciones más favorables a la creación de un estado de terror en la población objetivo, ya que puede beneficiarse de cierto grado de conocimiento que le aporte el grupo subyacente.

\subsection{La clasificación bifactorial del NSCITF}

Ahora que ya se ha analizado la clasificación dispuesta por Pantucci, resulta más sencillo comprender la catalogación bifactorial del terrorismo individual propuesta por el NSCITF (2015). Esto es así debido a que utilizando la variable de la conexión con el grupo ya manejada en el apartado anterior, se suma una variable adicional: la autonomía ideológica del grupo. Esto permite establecer una mayor diferenciación entre terroristas individuales que manifiestan el mismo nivel de conexión con un colectivo terrorista.

Así, las dos variables que se utilizarán para este estudio serán las siguientes: i) la competencia social del terrorista, es decir, su capacidad para actuar dentro de un grupo o mediante vinculaciones con el mismo, análoga a la anterior conexión con colectivos terroristas; y ii) la autonomía ideológica del atacante, esto es, la defensa o no de ideas y programas propios de un determinado grupo terrorista.

Como primer grupo de sujetos, se encuentran los lone followers, los cuales muestran niveles bajos de autonomía ideológica y de competencia social. Por esta razón, tienden a defender los programas y objetivos de un determinado colectivo terrorista. Sin embargo, su escasa competencia social les impide entrar a formar parte de este. 
En segundo lugar, con un alto nivel de competencia social, pero manteniendo un nivel bajo de autonomía ideológica, aparecen los lone soldiers. Estos defienden la misma ideología y objetivos que un colectivo terrorista y, aunque como terroristas individuales tienden a actuar en solitario, son los más proclives a establecer conexiones con grupos terroristas.

Tercero, en el lugar directamente opuesto a los lone soldiers, se encuentran los loners, los cuales gozan de un alto grado de autonomía ideológica y un bajo nivel de competencia social. Su diferencia ideológica respecto a los colectivos terroristas y sus problemas para actuar en grupo los llevan a actuar siempre en solitario.

Por último, ya mostrando niveles altos tanto de competencia social como de autonomía ideológica, aparecen los lone vanguards. Aunque disponen de una gran capacidad para el trabajo el grupo, su autonomía ideológica les impide actuar en el seno de un colectivo. Esta alta competencia social se manifiesta en la posibilidad de identificación con los valores de un grupo determinado, si bien, el lone vanguard suele aportar sus propios matices y desviaciones a dicha ideología.

Por todo ello, lo que en esencia realiza el NSCITF es una ampliación de la distinción dispuesta por Pantucci. Sin embargo, el hecho de que se añada una variable adicional provoca que los ejemplos que se determinan para el primero de los análisis no coincidan para el segundo de los mismos. Esto se percibe claramente en los ejemplos que ambos autores aportan para los loners y para los lone vanguards. Mientras que dentro de la primera diferenciación tanto el caso de Anders Breivik ${ }^{25}$ como el de Theodore Kaczynzski ${ }^{26}$ entran dentro de la categoría de loner (Pantucci, 2011: 14-19), en la segunda clasificación, el primero es incluido en los lone vanguards debido a su posibilidad de relación o influencia de y hacia terceros, y el segundo es catalogado como loner al no identificarse con ninguna opción concreta ni ideológica ni operacionalmente (NSCITF, 2015: 30-31).

Por otro lado, en cuanto a su peligrosidad, tanto los lone followers como los lone soldiers, gracias a reducida autonomía ideológica, muestran una mayor capacidad de crear un estado de terror entre la población, ya que sus acciones se identifican con las realizadas por un determinado colectivo terrorista. Análogamente, los loners son los que menos peligro representan debido a su reducido nivel de competencia social y su alto grado de autonomía ideológica. Finalmente, los lone vanguards merecen un análisis más detallado y enfocado al caso concreto puesto que muestran perfiles de liderazgo que podrían derivar en la ulterior formación de un colectivo.

\subsection{La clasificación multifactorial de Bates}

En último lugar, resulta también útil la clasificación postulada por Bates (2012). Esta amplía el número de variables utilizadas hasta cuatro, no coincidiendo ninguna de ellas con las utilizadas por Pantucci y el NSCITF. Sin embargo, esto no impide que, tras su análisis, se puedan identificar algunas similitudes entre las tres categorizaciones.

\footnotetext{
${ }^{25}$ Anders Breivik es el autor de la denominada Masacre de la Isla de Utoya y del atentado con bomba en el centro de Oslo, ambos perpetrados en fecha 22 de julio de 2011 y con un resultado de 79 muertos. Para conocer con mayor profundidad su ideología y su motivación para perpetrar estos dos ataques, véase BREIVIK (2011).

26 Theodore Kaczynski, más conocido como Unabomber, fue declarado autor del envío de 16 cartas bomba entre los años 1978 y 1995 con un resultado de 3 muertos y 23 heridos, todo ello como protesta contra el desarrollo tecnológico. Véase http://paralibros.com/passim/p20-soc/pq2095ub.htm.
} 
Bates hace referencia en primer lugar a la radicalización. Así, diferencia entre aquellos terroristas individuales que se han autorradicalizado, normalmente a través de internet, y los que han recibido instrucción y entrenamiento de parte de un colectivo o de un tercero terrorista. En segundo lugar, añade la variable de la motivación. Esta permite distinguir entre aquellos individuos que se proyectan por encima de la comunidad y promueven su propia ideología política de quienes se pliegan a los programas y objetivos de una determinada organización. Como tercera variable, hace referencia a la extensión de los actos del atacante, la cual permite establecer la diferencia entre el terrorista "de carrera", esto es, el que pretende atacar varias veces, y el terrorista caótico, a saber, el que pretende ejecutar un único y grave ataque. Finalmente, como última variable, plantea el grado de riesgo aceptable por el sujeto, siendo que, por un lado, se encuentra el terrorista averso al riesgo, el cual, normalmente, realizará ataques que permitan una huida más fácil; y, por otro lado, el terrorista amante del riesgo, que no otorga tanta importancia a poder huir del lugar del ataque.

En aplicación de dichas variables, el autor identifica cuatro posibles tipos de terrorista individual. En primer lugar, sitúa al lone wolf avenger, el cual se caracteriza por haberse autorradicalizado, no plegarse a los objetivos de un colectivo, tener una estrategia concreta a realizar en una pluralidad de ataques y ser contrario al riesgo. En este grupo se incluye el caso de Theodore Kaczynzski, ya que defendía su propia ideología, realiza una pluralidad de ataques y medía con exactitud la gravedad de los mismos para asegurarse no superar un determinado riesgo de ser capturado.

Con caracteres similares, a excepción del grado de aversión del riesgo, se encuentran los lone wolf vigilante. Al igual que el lone wolf avenger, muestra la defensa de unos objetivos propios adquiridos a través de la autorradicalización y desea realizar una serie de ataques, si bien, como diferencia, es amante del riesgo. Por esta razón, se muestra más dispuesto a realizar operaciones que puedan causar su muerte, al menos como eslabón final de su estrategia de atentados.

En tercer lugar, se encuentran los lone wolf revenger. Se caracterizan por haberse autorradicalizado y por defender unas ideas propias por encima de las de cualquier organización. Además, son más proclives a la realización de un acto extremadamente gravoso que a la perpetración de un conjunto de ataques en el marco de una estrategia concreta a medio o largo plazo. Asimismo, al igual que los lone wolf vigilante, son amantes del riesgo, con lo que no miden la gravedad del ataque con respecto a sus probabilidades de huida.

Como último tipo de terrorista individual, se encuentra el lone wolf guerrilla. Este es el único que no se ha autorradicalizado, pues ha recibido adiestramiento por parte de un colectivo terrorista. Coherentemente con ello, se siente identificado con los valores y objetivos de ese grupo y trabaja para su consecución. En el mismo sentido, no tolera un alto nivel de riesgo y es un terrorista de carrera, esto es, enmarca sus acciones en el ámbito de una estrategia terrorista proyectada más allá de un simple ataque.

Como se puede comprobar, esta última clasificación trata, al menos a grandes rasgos, la autonomía ideológica a través de la radicalización y la motivación. Además, dentro de estas dos variables también pueden percibirse ciertas referencias a la conexión con colectivos por cuanto si el terrorista pliega sus objetivos a los de una organización, normalmente, es más proclive a mantener contactos con la misma, si bien, esto no deja de ser un dato estadístico y no una relación causal. 
Sin embargo, el análisis de la estrategia del propio terrorista en cuanto a la pluralidad de sus potenciales acciones y de su grado de aversión al riesgo proporciona una visión adicional del mismo que abre la puerta a analizar de manera más concreta la peligrosidad de cada subtipo mencionado. A este respecto, el lone wolf guerrilla demuestra ser el más peligroso debido a su identificación con el programa y valores del grupo que le formó. Además, su aversión al riesgo y su proyección a largo plazo le convierten en una herramienta muy útil para un colectivo terrorista, al menos en lo que a la consecución de sus objetivos se refiere. Por debajo, en lo relativo al nivel de riesgo permisible, se encuentran los lone wolf revenger, superando a los lone wolf vigilante debido a su capacidad de continuidad en la perpetración de ataques. Finalmente, el menos peligroso de los cuatro es el lone wolf avenger por mostrar un perfil bajo, sin influencias externas y no hallarse dispuesto a correr muchos riesgos.

En conclusión, existen multitud de variables diferentes para clasificar a los terroristas individuales. En todo caso, desde el punto de vista penal, el único requisito para que dicha clasificación sea correcta es que se respete la proyección del terrorismo individual como diferencia respecto de la pertenencia a organizaciones y grupos terroristas.

Sin embargo, como ya se ha demostrado, para un conocimiento mayor del fenómeno del terrorismo individual es necesario incluir variables adicionales más allá del nivel de conexión con colectivos o terceros terroristas. Esto es así porque, según el terrorista defienda una ideología propia o la de una organización, según desee realizar una batería de atentados o únicamente uno solo, o incluso según busque sobrevivir al ataque o admita morir en el mismo, el estado de terror que pueda crear entre la población y, con ello, su peligrosidad intrínseca, será diferente.

\section{Conclusiones}

En esencia, desde el punto de vista jurídico, el terrorista individual es aquel sujeto que comete un delito que, tanto en su vertiente objetiva como subjetiva, cumple con los requisitos para ser considerado como un delito de terrorismo, pero sin que pueda considerarse perpetrado en el seno de una organización o grupo terrorista. Así, se concluye que podrán ser considerados terroristas individuales todos aquellos que actúen reiteradamente en solitario o en parejas, exceptuando quienes lo hagan únicamente por razones operativas, y aquellos que se integren en un colectivo que no muestre ninguna continuidad ni estructura.

Este examen realizado a la luz del terrorismo colectivo permite diferenciar entre los tres casos paradigmáticos que se incluyen dentro del fenómeno del terrorista comúnmente denominado "lobo solitario". En este sentido, se determina que tanto el terrorista individual propiamente dicho como el lobo solitario yihadista no pertenecen ni colaboran con un grupo u organización terrorista por lo que han de ser considerados terroristas individuales. Sin embargo, el terrorista individual táctico sí que cumple con los requisitos expresados por la Jurisprudencia para pertenecer a una organización por lo que se integra dentro del terrorismo colectivo.

Por último, mediante las clasificaciones examinadas se determina que el terrorista individual proyecta una mayor potencialidad lesiva cuanto más cercano a los objetivos de una organización se muestre, cuanta más influencia ostente un colectivo terrorista en su radicalización y entrenamiento y cuanto menos impulsivo se muestre en sus acciones. A través de las tres clasificaciones analizadas se aportan las herramientas para una futura evaluación detallada de la potencialidad lesiva del 
terrorismo individual y, con ello, de la legitimidad de su punición bajo la sistemática terrorista ${ }^{27}$.

\section{Bibliografía}

AGUILAR BLANC, C. (2012), "El terror de Estado francés: una perspectiva jurídica",

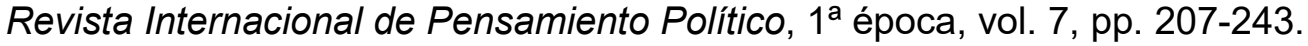

ASSOCIATED PRESS (2017), Covering tyranny. The AP and Nazi Germany: 19331945, disponible en la siguiente dirección electrónica:

https://www.ap.org/about/history/ap-in-germany-1933-1945/ap-in-germanyreport.pdf (fecha de consulta: 15 de junio de 2018).

AUDIENCIA NACIONAL 28/2000 de 10 de julio (RJ 4836/2000).

AUDIENCIA NACIONAL 70/2013 de 20 de diciembre (RJ 5522/2013).

BAKUNIN, M. (1870), Letters to a Frenchman on the Present Crisis, disponible en la siguiente dirección electrónica:

https://www.marxists.org/reference/archive/bakunin/works/1870/letterfrenchman.htm\#s1 (fecha de consulta: 3 de junio de 2018).

BATES, R. A. (2012), "Today's lone wolf terrorists. Dancing with wolves", The Journal of Public and Professional Sociology, vol. 4, issue 1, art. 1, disponible en la siguiente dirección electronica:

https://digitalcommons.kennesaw.edu/cgi/viewcontent.cgi?article=1023\&context =ipps (fecha de consulta: 22 de marzo de 2018).

BEAM, L. (1992), "Leaderless resistance", The Secesionist, núm. 12, disponible en la siguiente dirección electrónica: http://www.louisbeam.com/leaderless.htm (fecha de consulta: 10 de octubre de 2017).

BREIVIK, A. B. (2011), 2083: A European Declaration of Independence, disponible en la siguiente dirección electrónica:

https://archive.org/details/2083 A European Declaration of Independence (fecha de consulta: 21 de diciembre de 2017).

BURLEIGH, M. (2013), Sangre y rabia. Una historia cultural del terrorismo, Penguin Random House, Barcelona.

BURTON, F. y STEWART, S. (2008), "The Lone-Wolf Disconnect", Stratford, disponible en la siguiente dirección electrónica:

https://www.stratfor.com/weekly/lone wolf disconnect (fecha de consulta: 12 de febrero de 2018).

CANCIO MELIA, M. (2010), Los delitos de terrorismo: estructura típica e injusto, Reus, Madrid.

CAPELLA I ROIG, M. (2014), “¿Qué queda del delito político en el Derecho internacional contemporáneo? (Observaciones en los ámbitos de la extradición y el asilo)", Revista Electrónica de Estudios Internacionales, núm. 28, disponible en la siguiente dirección electrónica:

http://www.reei.org/index.php/revista/num28/articulos/queda-delito-politicoderecho-internacional-contemporaneo-observaciones-ambitos-extradicion-asilo (fecha de consulta: 3 de junio de 2018).

CAPITA REMEZAL, M. (2008), Análisis de la legislación penal antiterrorista, Colex, Madrid.

CASANOVA RUIZ, J. (2005), "Terror and violence. The dark face of Spanish anarchism", International Labor and Working-Class History, núm. 67, pp. 79-99.

\footnotetext{
${ }^{27}$ La crítica a la punición del terrorismo individual bajo la sistemática del terrorismo y no de la delincuencia común basada en la ausencia de potencialidad lesiva, es decir, en la imposibilidad de lesión del bien jurídico protegido por los delitos de terrorismo, ha sido realizada por diversos autores. Entre ellos, se pueden destacar Villegas Díaz (2016) y Tello Salinas (2016).
} 
CODEXTER (2015), Sub-Group on Terrorists Acting Alone, 29th plenary meeting, Estrasburgo, disponible en la siguiente dirección electrónica: https://rm.coe.int/16806b727e (fecha de consulta: 26 de febrero de 2018).

CRUICKSHANK, P. y ALI, M. H. (2007), "Abu Musab AI Suri: architect of the new AI Qaeda", Studies in Conflict \& Terrorism, núm. 30, pp. 1-14.

ETZENSBERGER, H. M. (2006), El corto verano de la anarquía. Vida y muerte de Durruti, Anagrama, Madrid.

EUROPOL (2016), "Lone actor attacks - Recent developments", European Counter Terrorism Centre, disponible en la siguiente dirección electrónica: https://www.europol.europa.eu/publications-documents/lone-actor-attacksrecent-developments (fecha de consulta: 25 de mayo de 2018).

GARCÍA MORIYÓN, F. (2017), "Asesinado por el anarquismo: anarquismo y violencia legítima”, Bajo Palabra. Revista de Filosofía, $2^{a}$ Época, núm. 15, pp. 117-134.

IVIANSKY, Z. (1977), "Individual terror: concept and typology", Journal of Contemporary History, núm. 12, pp. 43-63.

JIMÉNEZ DE ASÚA (1958), Principios de Derecho penal: la ley y el delito, Abeledo Perrot, $3^{\mathrm{a}}$ ed., Buenos Aires.

KINNEALLY III, J. J. (1987), "The political offence exception: Is the United StatesUnited Kingdom supplementary extradition treaty the beginning of the end?", American University International Law Review, vol. 2, issue 1, pp. 203-227.

KROPOTKIN, P. (1914), El espíritu de rebelión, trad. de Ed. Marginales, Ed. Marginales, disponible en la siguiente dirección electrónica: https://edicionesmarginales.files.wordpress.com/2016/11/espiritu-derebelion.pdf (fecha de consulta: 14 de mayo de 2018).

LAMARCA PÉREZ, C. (1985), Tratamiento jurídico del terrorismo, Centro de Publicaciones del Ministerio de Justicia, Colección Temas Penales, Madrid.

LAMPE, E. J. (2003), La dogmática jurídico-penal entre la ontología social y el funcionalismo, trad. de Carlos Gómez-Jara Díez, Grijley, Lima.

LENIN, V. I. (1902), ¿Qué hacer?, Alianza Editorial, Madrid.

MILLER, M. A. (1995), "The intellectual origins of modern terrorism in Europe". En: CRENSHAW, M., Terrorism in context, Filadelfia Penn State Press, pp. 27-62.

MONTORO BALLESTEROS, A. (2000), "En torno a la idea de delito político. (Notas para una ontología de los actos contrarios a Derecho)", Anales de Derecho, núm. 18, pp. 131-156.

NECHAEV, S. (1868), Catecismo de un revolucionario, disponible en la siguiente dirección electrónica: https://bibliotecanacionandaluzasevilla.files.wordpress.com/2008/10/catecismode-un-revolucionario.pdf (fecha de consulta: 26 de mayo de 2018).

NESSER, P. (2012), "Single actor terrorism: scope, characteristics and explanations", Perspectives on terrorism, vol. 6, núm. 6, pp. 61-73.

NSCITF (2015), "Report: Lone Wolf terrorism", Security Studies Program, Washington D. C., disponible en la siguiente dirección electrónica: http://georgetownsecuritystudiesreview.org/wp-content/uploads/2015/08/NCITFFinal-Paper.pdf (fecha de consulta: 13 de abril de 2018).

ORCHARD, P. (2014), A right to flee. Refugees, States and the Construction of International Cooperation, Cambridge University Press, Cambridge.

PANTUCCI, R. (2011), "A typology of lone wolves: preliminary analysis of lone Islamist terrorists", Developments in Radicalization and Political Violence, Londres, disponible en la siguiente dirección electronica:

https://www.trackingterrorism.org/system/files/chatter/1302002992ICSRPaper A TypologyofLoneWolves Pantucci.pdf (fecha de consulta: 21 de diciembre de 2017).

PERCEVAL VERDE, J. M. (2017), El terror y el terrorismo. Cómo la humanidad ha gestionado sus miedos, Cátedra, Madrid.

PÉREZ VENTURA, O. (2014), "Mustafá Setmarian, el ideólogo de la yihad moderna", 
Instituto Español de Estudios Estratégicos, núm. 5, disponible en la siguiente dirección electrónica:

http://www.ieee.es/Galerias/fichero/docs marco/2014/DIEEEM05-

2014 Mustafa Setmarian IdeologoYihadModerna OPVentura.pdf (fecha de consulta: 12 de febrero de 2018).

PRAKASH SINHA, S. (2013), Asylum and International Law, Martinus Nijhoff, La Haya.

PYLE, C. H. (2001), Extradition, Politics and Human Rights, Temple University Press, Philadelphia.

RAPOPORT, D. (2004), "The four waves of modern terrorism". En: KURTH, A. y LUDES, J.M. (eds.), Attacking terrorism: elements of a grand strategy, Washington D.C., Georgetown University Press, pp. 77-101.

ROBESPIERRE, M. (1794), Sobre los principios de moral política que deben guiar a la Convención Nacional en la administración interna de la República, disponible en la siguiente dirección electrónica:

https://www.marxists.org/espanol/tematica/cienpol/robespierre/moralpolitica.htm (fecha de consulta: 22 de mayo de 2018).

SPAAIJ, R. (2010), "The enigma of lone wolf terrorism. An assessment", Studies in conflict and terrorism, vol. 33, núm. 9, pp. 854-870.

TELLO SALINAS, F. (2016), "Terrorismo individual. Un enemigo imaginario. Un análisis crítico de los artículos núm. 1 y 2 de la Ley 18.314", Revista DIKÉ, vol. 1, Santiago de Chile pp. 83-106.

TOBOSO BUEZO, M. (2014a), "La contaminación del concepto Lobo Solitario", Revista Catalana de Seguretat Pública, núm. 17, pp. 6-26.

TOBOSO BUEZO, M. (2014b), Lobos de Occidente. El terrorismo individual como elemento emergente y evolución táctica de Al-Qaeda, Instituto Universitario General Gutiérrez Mellado, Madrid.

TOBOSO BUEZO, M. (2017), "2016: escenario negro, ¿en regresión?", Análisis GESI, núm. 1/2017, disponible en la siguiente dirección electrónica:

http://www.seguridadinternacional.es/?q=es/content/2016-escenarionegro\%E2\%80\%A6-\%C2\%BFen-regresi\%C3\%B3n (fecha de consulta: 17 de octubre de 2018).

TRIBUNAL SUPREMO 886/2007 de 2 de noviembre (RJ 10272/2007).

TRIBUNAL SUPREMO 309/2013 de 1 de abril (RJ 11151/2012).

TRIBUNAL SUPREMO 719/2013 de 9 de octubre (RJ 10566/2013).

TRIBUNAL SUPREMO 271/2014 de 25 de marzo (RJ 10892/2014).

TRIBUNAL SUPREMO 289/2014 de 8 de abril (RJ 2158/2013).

TRIBUNAL SUPREMO 445/2014 de 29 de mayo (RJ 11106/2013).

TRIBUNAL SUPREMO 577/2014 de 12 de julio (RJ 10071/2014).

TRIBUNAL SUPREMO 576/2014 de 18 de julio (RJ 10028/2014).

TRIBUNAL SUPREMO 277/2016 de 6 de abril (RJ 10714/2015).

TRIBUNAL SUPREMO 714/2016 de 26 de septiembre (RJ 1951/2015).

TRIBUNAL SUPREMO 798/2016 de 25 de octubre (RJ 10224/2016).

TRIBUNAL SUPREMO 607/2017 de 15 de septiembre (RJ 10792/2016).

TROTSKY, L. (1914), La posición marxista acerca del terrorismo individual, disponible en la siguiente dirección electrónica:

https://rosaluxemburgo.files.wordpress.com/2009/04/trostky3.pdf (fecha de consulta: 22 de abril de 2018).

TROTSKY, L. (1935), Terrorismo y comunismo, Fundación Federico Engels, Madrid.

VILLEGAS DÍAZ, M. (2016), "La tentativa inidónea en los delitos de terrorismo en el Derecho penal chileno", Revista de Ciencias Penales, 6a época, vol. XLIII, núm. 3, pp. 13-32. 